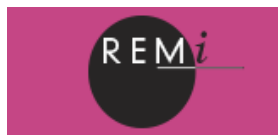

Revue européenne des migrations internationales

vol. 28 - $n^{\circ} 4 \mid 2012$

Migration et confection

\title{
Travailleurs migrants du prêt-à-porter en Chine. Flexibilités et opportunités
}

Migrant Workers and the Garment Industry in China. Flexibilities and

Opportunities

Trabajadores migrantes del pronto a vestir en China. Flexibilidades y

oportunidades

\section{Gilles Guiheux}

\section{(Q) OpenEdition}

Journals

Édition électronique

URL : https://journals.openedition.org/remi/6198

DOI : 10.4000/remi.6198

ISSN : $1777-5418$

Éditeur

Université de Poitiers

Édition imprimée

Date de publication : 1 décembre 2012

Pagination : 27-42

ISBN : 979-10-90426-06-1

ISSN : 0765-0752

\section{Référence électronique}

Gilles Guiheux, "Travailleurs migrants du prêt-à-porter en Chine. Flexibilités et opportunités », Revue européenne des migrations internationales [En ligne], vol. 28 - n4 | 2012, mis en ligne le 01 décembre 2015, consulté le 16 avril 2022. URL : http://journals.openedition.org/remi/6198 ; DOI : https://doi.org/ 10.4000/remi.6198 


\section{Travailleurs migrants du prêt-à-porter en Chine. Flexibilités et opportunités}

\section{Gilles Guiheux ${ }^{1}$}

Les industries textiles ont connu des mutations majeures au cours des dernières décennies. Des pans entiers de l'appareil industriel des pays du Nord ont été démantelés pour être délocalisés dans des pays à bas coût de maind'œuvre en Asie, en Afrique du Nord ou en Europe de I'Est. La Chine, en particulier, est devenue l'un des premiers centres de production. Pourtant, l'industrie $y$ est loin d'être homogène. Les usines employant des milliers de travailleurs selon des méthodes de production fordistes pour le marché national et international constituent la face la plus connue de l'industrie chinoise du prêt-à-porter. $C^{\prime}$ est une production de masse organisée dans le cadre de plusieurs échelons de sous-traitance, le premier donneur d'ordre, souvent étranger, s'appuie sur une main-d'œuvre nombreuse et mal rémunérée, souvent féminine et d'origine rurale. Mais la Chine compte aussi des créateurs qui fabriquent de petites séries dans des conditions artisanales et dont les vêtements défilent sur les podiums des capitales mondiales de la mode. Enfin, toute une partie de la population rurale continue de faire confectionner ses vêtements par des tailleurs, dont les instruments de travail sont le ciseau et la machine à coudre. Les modes d'organisation de la production et le recours à telle ou telle technologie dépendent du marché visé - rural ou urbain, national ou international, à pouvoir d'achat faible, moyen ou élevé - et de la province concernée - la Chine côtière tournée vers l'exportation, les villes capitales de Pékin et Shanghai qui lancent les modes, ou la Chine rurale de l'intérieur².

L'industrie de la confection a fait l'objet de nombreux travaux qui se sont intéressés aux conditions de production dans les capitales de la création - Paris, New York, Londres, etc. Ils ont montré notamment que l'industrie du vêtement se caractérisait par des variations saisonnières et d'incessants changements de mode et que les entrepreneurs devaient combiner flexibilité et standardisation. Ces contraintes ont conféré des conditions particulières au recrutement de la main-d'œuvre et aux conditions de travail : faibles qualifications, bas salaires, sous-traitance, saisonnalité (Green, 1998). D'autres recherches ont permis de comprendre combien partout le dynamisme du secteur a reposé sur le recru-

1 Professeur des Universités, Université Paris Diderot, Sedet ; gilles.guiheux@univ-parisdiderot.fr

2 Cette diversité des conditions de fabrication du prêt-à-porter en Chine est le sujet d'un film mélangeant fiction et documentaire, Wu Yong (Useless), du réalisateur Jia Zhangke (2007). 
tement d'une main-d'œuvre de migrants internationaux primo-arrivants ou de femmes migrantes internes, prêts à accepter la flexibilité et la précarité des conditions de travail (Green, 1998 ; Chin, 2005). Elles ont aussi mis en évidence combien le secteur de la confection constituait une source d'opportunités pour les immigrants les moins qualifiés; les trajectoires professionnelles ascendantes y étaient plus probables que dans d'autres secteurs d'activité, comme les services domestiques par exemple. Le prêt-à-porter apparaît comme un secteur d'activité où il est possible de faire aisément l'acquisition de compétences professionnelles - maîtrise d'un savoir-faire, d'un réseau de fournisseurs ou de clients. Dans le cadre de réseaux de sous-traitance sans cesse renouvelés et alors que le capital nécessaire à la création d'une entreprise est minime, cela autorise des parcours socio-professionnels ascendants (Waldinger, 1984 et 1986). Enfin, des travaux se sont intéressés à la dimension d'entrepreneuriat ethnique de l'industrie de la confection, montrant comment celle-ci permettait à des patrons d'intégrer leur parentèle ou d'autres migrants de la même origine (Waldinger, 1986 ; Zhou, 1992).

À la lumière de ces conclusions tirées de terrains européens et nord-américains, cet article entend questionner les conditions de production du prêt-à-porter en Chine et la place qu'y occupent les travailleurs migrants. II ne s'agit pas là de migrants internationaux mais bien de migrants internes venus des provinces rurales pauvres où la main-d'œuvre est largement surnuméraire. Ils partagent avec les premiers un certain nombre de caractéristiques liées non seulement à leur désir d'ascension sociale - attirés par le mirage urbain, ils quittent les campagnes pour fuir la pauvreté -, mais aussi aux discriminations dont ils sont victimes. En effet, bien qu'assoupli, le système d'enregistrement de la population et du certificat de résidence mis en place en 1958 pour servir le projet socialiste de développement ${ }^{3}$, le hukou, se maintient et limite l'accès des populations migrantes à toute une série de biens et services - santé, éducation, logement, etc. - ; les populations migrantes sont en Chine, comme les immigrants dans les pays du Nord, des citoyens de seconde catégorie. Comme l'ont montré sur $\mathrm{d}^{\prime}$ 'autres terrains ${ }^{4}$ les recherches de Chin notamment (2005), notre hypothèse est que, bien qu'en Chine comme ailleurs, les travailleurs migrants du prêt-à-porter travaillent dans des conditions difficiles pour des salaires faibles, ils ne sont pas simplement les victimes passives d'un système d'emploi oppressif.

Cette contribution s'appuie sur la trajectoire biographique et professionnelle des membres d'une fratrie, rencontrée à Yiwu, ville marchande et manufactu-

\footnotetext{
3 Le hukou s'inspire directement du système de la propiska instauré en URSS en 1932 ; comme lui, il est un instrument de planification, de redistribution des ressources et de contrôle des migrations. Pendant la période maoïste, le système du hukou a empêché toute mobilité des campagnes vers les villes, permettant de drainer des centaines de milliards de yuans des premières vers les secondes et induisant ainsi une industrialisation sans urbanisation. Réformé, le hukou reste un instrument fondamental de gestion du développement et de contrôle social, au service de deux priorités : maintenir la stabilité sociale et un fort taux de croissance. Les travailleurs migrants (mingong) restent des paysans de statut quand bien même ils vivent et travaillent en ville depuis plusieurs années (Froissart, 2008).

4 Selon Margaret M. Chin, les travailleurs immigrés de la confection new-yorkaise ne cherchent pas les emplois les mieux rémunérés, mais " favor jobs that complement their household roles as parents, providers, or supporters of relatives overseas " (Chin 2005 : 2). Elle montre en particulier que la flexibilité des heures de travail présente des avantages pour les mères de famille.
} 
rière de la province du Zhejiang, au sud de Shanghai. Nés à la fin des années 1960 et au début de la décennie 1970, deux sœurs et un frère travaillent depuis vingt ans dans l'industrie locale du prêt-à-porter. Leurs parcours socio-professionnels seront rapportés aux caractéristiques du secteur fait d'une multitude de petites et moyennes entreprises travaillant à la fois en sous-traitance et en concurrence les unes avec les autres. Les établissements sont de taille variable et comptent moins de dix employés parfois, plusieurs dizaines pour d'autres. À Yiwu, l'industrie de la confection correspond bien à la définition qu'en donne Nancy Green : " une industrie de production de masse sans méthodes classiques de production de masse "(1998: 16). On montrera que dans cette ville, la flexibilité est source d'opportunités. Les matériaux utilisés ont été rassemblés au cours de plusieurs enquêtes de terrains conduites sur place depuis 2006, des entretiens biographiques réalisés en septembre 2009 avec les trois personnages principaux ${ }^{5}$ et des articles parus dans la presse locale.

\section{Yiwu, place marchande et manufacturière, terre d'accueil de travailleurs de l'extérieur}

Yiwu, située au centre de la province du Zhejiang, au sud de Shanghai, est d'abord une place marchande spécialisée dans la vente en gros d'articles manufacturés destinés aux consommateurs chinois ou étrangers - le terme utilisé localement est celui de marché "des petits articles " (xiaoshangpin ou small commodities). Cette spécialisation tient d'une part à trois décennies d'initiatives prises par le gouvernement local pour mettre à la disposition de commerçants les espaces nécessaires à l'exposition de leurs produits et à la mise en place d'instances de régulation de l'activité commerciale. Elle s'explique d'autre part par le besoin, pour la multitude de petites et moyennes entreprises familiales de la Chine méridionale et côtière, de disposer de canaux de distribution de leur production. Aujourd'hui, la place marchande est située pour l'essentiel dans des infrastructures modernes situées au nord de la ville et qui s'apparentent à un centre permanent d'exposition. Celui-ci couvre 2,6 millions de mètres carrés, compte 58000 stands, offre aux acheteurs 400000 produits différents classés dans 1900 catégories (Guiheux, 2011). Une part importante de cette activité commerciale porte sur des articles de prêt-à-porter. Autrefois installé en centreville dans un bâtiment édifié en 1993, le marché de la confection a déménagé en 2002 dans ce complexe moderne (Xin huangyuan, etc., 2011). Les stands sont passés d'une surface de $2,8 \mathrm{~m}^{2}$ à $14,4 \mathrm{~m}^{2}$. Plus de 5000 stands sont installés sur huit niveaux et regroupés par catégorie : articles en jean au premier niveau, vêtements en cuir au second, confection pour femmes au troisième, pyjamas, vêtements de laine et de sport au quatrième, vêtements d'enfants au cinquième, etc. Au total, les articles textiles représentent, derrière les articles électriques, le second poste des exportations de la ville d'Yiwu, soit $14 \%$ du total en $2011^{6}$. Les

\footnotetext{
5 Les entretiens ont été réalisés à Yiwu les 11, 14 et 15 septembre 2009. Toutes les informations relatives aux trois informateurs figurant dans le texte, et dont les noms ont été modifiés, proviennent de ces entretiens. Ils ont été réalisés conjointement avec Bernard Ganne (Centre Max Weber, Lyon) et Shi Lu (IAO, Lyon). Notre séjour avait été rendu possible par une invitation des professeurs Jin Xiangrong et Zhu Xiwei (Center for Research of Private Economy, Université du Zhejiang) qui sont ici remerciés.

6 Statistiques des douanes de la ville de Yiwu, [en ligne] consultées le 26/04/2012.

URL : http://big5.ocn.com.cn/free/201202/fuzhuang061524.shtml
} 
produits disponibles sont des produits très bon marché et bas de gamme; sur le marché intérieur, ils sont destinés à la clientèle rurale, à l'exportation - $55 \%$ des transactions commerciales réalisées ( $M u, 2010)$-, ils sont destinés aux pays du Sud : Asie du Sud, Moyen-Orient, Afrique. À cet égard, Yiwu constitue une place marchande où s'approvisionnent les pauvres du monde entier.

L'activité commerciale se double d'une activité industrielle. De nombreuses petites et moyennes entreprises ont été créées ou sont venues s'installer sur place au fur et à mesure que se développait la place commerciale. 16000 entreprises industrielles sont enregistrées dans la ville d'Yiwu (Mu, 2010). Pour certains produits, la production locale représente une part majeure de la production nationale : $65 \%$ de la production de la bijouterie fantaisie, $35 \%$ des bas et collants, plus de $30 \%$ des fermetures à glissières. L'activité productive se fait selon des modalités diverses. Elle est réalisée pour partie dans de grandes usines qui emploient des centaines ou des milliers de salariés. À cette production de masse s'ajoutent des ateliers de production situés dans des zones urbaines et rurales de taille variable. Un même atelier peut compter plusieurs dizaines de salariés en période de forte activité et être réduit à quelques-uns lorsque les commandes sont en baisse. Une partie de la production est réalisée à façon par des familles qui travaillent à domicile. Ce sont ces petits et moyens producteurs qui ont le plus besoin du marché d'Yiwu comme vitrine de leur production, tandis que les plus grandes entreprises traitent directement avec leurs clients nationaux ou étrangers.

Cette économie commerciale et manufacturière s'appuie en grande partie sur une main-d'œuvre migrante. Début 2012, celle-ci s'élève à 1,5 million de personnes, soit deux fois la population enregistrée comme locale, c'est-à-dire dont le certificat d'enregistrement (le hukou) porte la mention Yiwu (Yiwu wailai renkou, etc., 2012). Cette distinction - les statistiques officielles comptabilisaient 230 millions de migrants dans tout le pays fin 2011 - est le fruit du système d'enregistrement de la population mis en place en 1958 et qui visait à empêcher alors tout exode rural. Depuis 1990, I'assouplissement du système a permis des migrations massives vers les villes mais les prérequis à l'obtention du hukou urbain restent en vigueur et les grandes villes ont maintenu un dispositif discriminatoire à l'encontre des migrants. Le rapport entre population locale et population allogène à Yiwu (un à deux) n'est pas exceptionnel dans des bourgs autrefois ruraux ; c'est en particulier le cas dans les zones rurales industrialisées des provinces du Guangdong et du Zhejiang qui ont massivement fait appel à des travailleurs venus d'ailleurs. À cet égard, I'usage du terme " migrant " est impropre car ces travailleurs peuvent être installés depuis plusieurs années avec conjoints et enfants et ne plus envisager ni de retourner dans leur village $\mathrm{d}^{\prime}$ origine ni de tenter leur chance dans une autre province - ce que suggère pourtant l'expression chinoise de "population flottante " (liudong renkou). II est donc plus juste de les désigner par l'expression de " population venue de l'extérieur "(wailai renkou). Malgré les réformes, leurs droits sociaux et économiques - accès aux services de santé, d'éducation et au logement - sont toujours moindres que ceux des résidents enregistrés localement. Bien qu'assoupli et en débat, ce système de citoyennetés plurielles, qui a assuré une maind'œuvre nombreuse et bon marché aux entreprises et qui est donc indissociable de la stratégie de développement économique suivi, semble devoir durer (Chan, 2010). 
À Yiwu, les plus gros contingents de travailleurs de l'extérieur proviennent de la province voisine du Jiangxi (270 000), de la province méridionale du Guizhou (196 000) et de la province centrale du Hunan (175 000) (Yiwu wailai, etc., 2012). Le gouvernement local s'enorgueillit d'une politique particulièrement progressiste à l'égard de ces populations, qualifiées depuis quelques années de " nouveaux habitants d'Yiwu " (xin Yiwu ren), expression qui manifeste clairement une intention d'intégration. De fait, de nombreuses initiatives ont été prises pour faciliter leur accueil et leur installation. Depuis 2001, des représentants des migrants participent aux assemblées locales, bien avant que cela ne devienne le cas au niveau national. En 2003 s'ouvre une bourse du travail de 4000 mètres carrés où affluent quotidiennement des centaines de travailleurs demandeurs d'emploi et des employeurs à la recherche de main-d'œuvre. Depuis 2004, pour mieux encadrer les flux de travailleurs, des accords ont été signés entre la municipalité et des gouvernements provinciaux ou municipaux de sorte qu'avant même d'arriver à Yiwu, les travailleurs sont informés des emplois à pourvoir. Plus récemment enfin, en 2011, une administration spécifique de contrôle et de service a été mise sur pied : le bureau de Gestion et de service auprès de la population migrante de la municipalité d'Yiwu (Yiwushi liudong renkou fuwu guanli ju) (Waidiren de, etc., 2011). De manière générale, comparativement à d'autres, le Zhejiang comme le Guangdong sont des provinces où l'assouplissement du système du hukou est le plus avancé ; depuis 2012, I'accès aux services publics d'éducation et de santé pour les travailleurs de l'extérieur y est grandement facilité (Li, 2012).

Cette contribution prend appui sur la trajectoire professionnelle et personnelle de deux sœurs et un frère qui s'installent successivement à Yiwu entre 1985 et 1994. Les deux aînées, Wang Ling, née en 1968 et Wang Yan, née en 1970, s'établissent à Yiwu en 1985 et 1988 ; elles ont respectivement dix-sept et dix-huit ans. Leur frère, Wang Jun, né en 1975, arrive en 1991 ; il a alors seize ans. Lors des entretiens réalisés en septembre 2009, les deux sœurs tiennent des commerces de vêtements sur le marché de gros d'Yiwu tandis que leur frère est à la tête d'une entreprise de confection de foulards qui emploie une centaine de personnes. Tous les trois sont nés et ont grandi dans la province voisine pauvre du Jiangxi, dans le district de Yiyang, dans la préfecture de Shanghrao frontalière du Zhejiang, à environ 300 kilomètres d'Yiwu. Leur ville natale est aujourd'hui reliée à Yiwu par le chemin de fer et par une autoroute.

Le choix de cette fratrie comme point d'appui de cette contribution s'explique par la qualité de l'information recueillie. Le frère est d'ailleurs une personnalité quasi publique par sa qualité de migrant ayant réussi. La presse locale, à Yiwu et dans son district d'origine, a ainsi publié plusieurs articles louangeurs vantant ses mérites personnels (Wang, 2009 ; Wu, 2007). Aux yeux des médias, qui servent pour partie de relais à la politique du Parti communiste, sa trajectoire est emblématique de ces héros de la Chine contemporaine qui s'appuient sur leurs propres forces pour créer de la richesse et des emplois (Guiheux, 2009). L'un de ces articles, par exemple, se conclut ainsi : "On peut dire que la raison du succès de [Wang Jun], c'est son courage et son énergie. Son esprit d'innovation doit faire un objet d'étude pour les entrepreneurs [...]. Nous croyons que I'histoire de [Wang Jun] constitue une source utile d'inspiration " (Wang, 2009). À la différence de cette littérature de propagande qui vise à inciter les travailleurs à compter uniquement sur leurs propres forces morales - " courage ", " énergie ", 
" esprit d'innovation " - pour se hisser dans la hiérarchie sociale, notre contribution entend rapporter la réussite de cet entrepreneur du prêt-à-porter aux conditions économiques et sociologiques de l'exercice de sa profession.

\section{La famille, ressource pour les migrants}

Les ressources familiales peuvent être considérées comme une explication principale de la destination de la migration de cette fratrie. Tous trois donnent plusieurs motifs à leur départ. La première à partir, Wang Ling, évoque I'environnement social et économique de l'époque. Dans leur village, au milieu des années 1980 - les communes populaires viennent d'être démantelées -, la propagande, encore diffusée par haut-parleurs, vante tous les jours les familles rurales récemment enrichies, alors qualifiées de "familles aux 10000 yuans" (wanyuan hu). Cela, dit-elle, l'a incitée à vouloir sortir de la pauvreté rurale. Tous les trois invoquent également leurs mauvais résultats scolaires et leur peu d'appétence pour les études; de fait ils quittent tous leur village avant d'avoir achevé leur scolarité secondaire. La migration est une alternative à la réussite scolaire pour satisfaire un désir d'ascension sociale. Sont également mentionnées des disputes familiales ; c'est en particulier le cas de l'aînée qui évoque une mésentente pérenne avec sa sœur cadette et la nécessité pour elle de quitter la cellule familiale conflictuelle.

Deux autres éléments biographiques contribuent à expliquer la migration. Le premier tient à I'histoire migratoire de la famille. Leurs parents installés à Yiyang dans le Jiangxi n'en sont pourtant pas originaires. Le père est né dans la province voisine du Hunan et son installation à la campagne date du mouvement des Jeunes instruits durant lequel les diplômés du lycée et de l'université sont envoyés dans les campagnes pour être rééduqués. Le père, qui a fait une scolarité secondaire complète, n'est donc pas un authentique paysan, mais a été victime d'une mobilité forcée. Leur mère est originaire de Yiwu, et $c^{\prime}$ est donc leur famille maternelle, oncles et tantes, qui les accueillent à leur arrivée dans le Zhejiang. Lorsque les deux aînées rejoignent Yiwu, celle-ci n'est pas encore devenue la ville prospère et moderne qu'elle est aujourd'hui ; c'est un gros bourg rural dont les activités marchandes sont en plein développement. La particularité de cette migration est que la fratrie Wang quitte une campagne pauvre non pas pour une ville côtière à l'avant-garde des réformes - Canton ou Shenzhen -, mais pour une campagne en cours d'industrialisation sur la base d'ateliers familiaux de production.

Lorsque Wang Ling arrive à Yiwu, elle travaille pendant huit mois dans un atelier de production tenu par un oncle qui fabrique des brosses en poil de porc. Lorsque sa sœur Wang Yan arrive à Yiwu quelques années plus tard, elle est logée par une tante enseignante, et confectionne des vêtements en sous-traitance à domicile ; $c^{\prime}$ est alors sa première expérience dans le prêt-à-porter alors qu'elle n'a aucune formation. Wang Jun est le seul de la fratrie à ne pas avoir recours à sa parentèle, bien que logé par ses sœurs, pour trouver son premier emploi ; à son arrivée, il suit une formation d'une semaine où il apprend à coudre les ourlets à la machine. II est ensuite embauché dans une usine après avoir répondu à une annonce. II nous dit d'ailleurs, lors de l'entretien, qu'il était très facile de trouver du travail au début des années 1990. 
La famille reste une ressource importante non seulement au moment de l'arrivée, mais tout au long de la vie professionnelle. Nos trois itinéraires en offrent de multiples exemples. Le mariage représente ainsi une étape non seulement dans la biographie des individus mais aussi dans le déroulement de leur carrière quand le rassemblement de capitaux économiques et relationnels permet la mise à son compte. Après son mariage, Wang Yan et son mari, qui exerce le métier de tailleur, vont monter un atelier de confection. La production démarre dans l'espace domestique, puis au bout de deux ans, le couple loue un local, achète des machines et fait venir des ouvriers du village d'origine de Wang Yan, recrutés parmi les membres de la famille et les voisins. Ils sont alors une dizaine de personnes à travailler dans I'atelier. Quant à son frère, Wang Jun, $c^{\prime}$ est avec un cousin qu'il s'associe pour ouvrir un commerce de foulards au sein du marché de gros, en 2003. Après l'échec de ce premier projet, il va travailler avec sa femme. Elle tient un stand sur le marché de gros et lui prend en charge l'atelier de production; pour créer son entreprise, il a emprunté 50000 yuans à sa sœur aînée (Wang, 2009). Wang Ling enfin, sa sœur aînée, a recours à deux reprises à un emprunt auprès de ses parents pour se lancer dans l'activité commerciale : en 2003, lorsqu'elle loue un stand dans le marché aux chaussettes de Yiwu (sa mère lui prête 8000 yuans) et à nouveau trois ans plus tard, lorsqu'elle commence à faire le commerce de jeans au marché du prêt-à-porter de Binwang (elle emprunte alors 20000 yuans également à sa mère). La famille peut être une solution au problème de l'accumulation initiale du capital, tout comme elle peut permettre de rassembler les ressources humaines nécessaires au lancement d'une activité indépendante, dans une économie dominée par les petites et moyennes entreprises. Celles-ci sont en grande majorité familiales et les entreprises créées par les migrants n'échappent pas à cette caractéristique (Guiheux, 2005). En retour, l'intervention de famille crée des obligations. C'est ainsi qu'en 2009, Wang Jun va employer le mari de sa sœur qui travaillait à domicile à l'emballage de la production de foulards et son neveu comme chauffeur.

Mais la famille peut être aussi une source de conflits. Wang Jun explique que ses parents se sont opposés à ce qu'il quitte son emploi d'ouvrier dans une usine pour s'établir à son propre compte et lorsque l'entreprise commerciale qu'il avait créée avec son cousin a cessé son activité, il dit s'être séparé de son parent à la suite de désaccords sur la stratégie à mener concernant le type de produit à fabriquer et les modes de commercialisation à adopter - fallait-il ou non conserver le stand sur le marché d'Yiwu ? Le fait d'employer des membres de sa propre famille n'est pas non plus exempt de risques. Wang Yan relate qu'elle a formé huit membres de sa famille qui ont travaillé pour le stand de vêtements qu'elle loue sur le marché, mais ceux-ci n'ont pas toujours fait preuve de loyauté. Ainsi parle-t-elle de sa sœur : " quand je n'étais pas là, ma sœur donnait sa propre carte de visite et pas la mienne ". De sorte qu'elle lui a ainsi volé une partie de sa clientèle. Même si elle lui a " tout appris " selon elle, la concurrence entre les membres d'une même famille reste vive.

Au-delà de la famille, le lien avec le village d'origine est bien maintenu. II approvisionne les entreprises d'Yiwu en main-d'œuvre mais aussi en produits. Wang Yan, par exemple quand elle s'est mise à son compte a fait venir quatre personnes de son village pour travailler dans son atelier de confection et en 2009, le commerce qu'elle tient sur le marché d'Yiwu s'approvisionne pour 
partie dans la province d'origine de son mari. Elle compte aussi parmi ses fournisseurs, un beau-frère et ses beaux-parents.

Les données que nous avons recueillies sur les itinéraires biographiques et professionnels de cette fratrie ne permettent cependant pas de conclure clairement à l'existence, à Yiwu, d'une communauté de migrants originaires du Jiangxi au sein de laquelle circuleraient des ressources et s'échangeraient des solidarités, comme le suggère la littérature sur l'entrepreneuriat ethnique. Dans le contexte chinois, l'identité provinciale est aussi linguistique ; appartenir à une communauté de gens de la même origine géographique, c'est d'abord parler la même langue, distincte de celle du lieu d'accueil. Wang Jun témoigne néanmoins qu'après avoir travaillé six mois à Yiwu, il a quitté la ville pour la capitale provinciale Hangzhou avec d'autres travailleurs originaires du Jiangxi ; il précise même que les liens qu'ils partagent avec ses camarades ont été forgés à Yiwu et qu'il ne s'agit pas de connaissances antérieures à sa migration. Sans doute le sentiment d'appartenance et le recours à sa communauté pour des activités entrepreneuriales varie-t-il en fonction des circonstances. Ainsi, aujourd'hui, les gouvernements locaux de régions pauvres incitent ouvertement leurs migrants à revenir investir dans leur lieu d'origine dans l'objectif de contribuer au développement de leur communauté. Un article de presse par exemple fait référence à une délégation partie d'Yiyang au printemps 2010 à destination de la province du Zhejiang, des villes de Dongyang, Yiwu, Hangzhou et de Shanghai pour trouver des investisseurs. Wang Jun, qualifié d' " homme qui est né et a grandi sur la terre de Yiyang "(tusheng tuzhang de Yiyangren), répond en ces termes au journaliste qui l'interroge sur la possibilité d'investissement dans sa région d'origine : "La raison pour laquelle nous aimerions revenir pour participer au développement de notre pays natal, c'est que par rapport aux gens de l'extérieur, nous disposons d'avantages et nous avons davantage confiance dans le développement économique de notre pays " (Yiyang xian zhaoshang, etc., 2010). D'autres articles récents témoignent de cette stratégie des gouvernements locaux (Yiyang xian tuijin, etc., 2011 ; Zheng Guangquan, etc., 2011).

\section{Les formes de la flexibilité}

Les carrières professionnelles de nos trois informateurs sont marquées par de multiples tournants qui s'expliquent aussi bien par des effets de la conjoncture économique - baisse ou hausse de l'activité -, de nouvelles opportunités débouchés ou sources d'approvisionnements -, que de situations personnelles problèmes de santé ou changement de situation familiale (mariage ou séparation). Précarité et flexibilité prennent ainsi la forme de l'alternance fréquente entre emploi salarié et travail indépendant - chemin qui peut être emprunté dans les deux sens à plusieurs reprises au cours d'une vie professionnelle -, de l'alternance entre activité manufacturière et activité commerciale, du passage d'un produit à un autre, ou encore de la combinaison entre production et commande à des sous-traitants.

En 1993, après deux années comme salarié d'une entreprise textile à Hangzhou, Wang Jun rentre à Yiwu ; face aux difficultés à trouver un emploi, il se met alors à son propre compte à domicile où il installe deux machines. II fabrique jusqu'à soixante chemises par jour, travaillant comme sous-traitant 
d'un donneur d'ordre. Après quelques mois, alors que ses revenus sont pourtant relativement confortables (1 600 yuans par mois), il décide de quitter cette activité. Contre l'avis de ses parents, il cherche à s'employer comme tailleur salarié dans une usine par souci d'apprendre de nouvelles techniques. Au bout de deux ans, il sait fabriquer des patrons et se met à nouveau à son compte. II travaille alors pour plusieurs clients qui lui fournissent des chemises à copier ; il fabrique le patron pour l'atelier qui aura la charge de la production. D'abord seul, il travaille rapidement avec des apprentis et l'atelier compte jusqu'à une vingtaine de personnes. En 2003, il se lance dans la production de foulards son activité en 2009 lors de l'entretien -, en association avec son cousin : il loue un atelier, achète six machines et emploie une dizaine de personnes. Parmi les raisons qu'il donne pour expliquer son changement de situation, il mentionne le fait qu'il est désormais propriétaire d'un appartement et qu'il s'est marié.

Dans le parcours de ses sœurs (voir supra), ce qui frappe est l'alternance répétée entre activité manufacturière et activité commerciale. En 1988, après avoir confectionné des vêtements à son domicile et recruté quatre personnes venues de son village, Wang Ling va, neuf ans plus tard, avoir suffisamment accumulé de capital pour acheter un appartement dans le Jiangxi. Elle retourne $s^{\prime} y$ installer et forte de l'expérience acquise, elle ouvre un commerce de détail où elle vend des vêtements fabriqués àYiwu? . Mais les dividendes ne sont guère élevés et une fois son capital épuisé, elle revient à Yiwu pour chercher un nouvel emploi ; elle ne quittera plus la ville. En 1998, elle est employée à un poste de contrôle qualité puis devient chef d'atelier, rémunérée 1300 yuans par mois. Les conditions sont, juge-t-elle, difficiles - travail pénible et horaires trop longs - et elle démissionne au bout d'un an. Elle reprend une activité commerciale avec l'aide de sa mère, loue un stand sur le marché de gros d'Yiwu et vend des articles de confection féminine. Victime d'un client malhonnête - elle perd plusieurs milliers de yuans -, elle se met alors à travailler pour le compte de sa sœur et de son frère. En 2006, après trois ans comme employée, elle s'établit à nouveau à son compte.

Sa sœur Wang Yan travaille successivement comme ouvrière, puis s'établit à son compte en ouvrant un atelier de confection, puis cesse cette activité pour faire du commerce. Arrivée à Yiwu, elle est d'abord employée dans l'atelier de fabrication de son oncle, puis dans un atelier de fabrication de vêtements. Après son mariage, le couple ouvre un atelier de confection, puis l'affaire est liquidée et les machines vendues. Elle va par la suite ouvrir un commerce de vêtements d'enfants. La marchandise est achetée à Hangzhou et revendue à Yiwu où la fabrication locale est de moindre qualité. Enfin, elle tiendra un stand sur le marché de gros d'Yiwu. Pour survivre face à une vive concurrence, elle renouvelle souvent ses produits. En 2009, elle achète ses nouveaux modèles à Canton, qu'elle fait ensuite reproduire dans des ateliers du Hunan où la main-d'œuvre est moins chère et le produit de moindre qualité.

Une autre dimension de la flexibilité concerne le passage d'un produit à un autre. Lorsqu'en 2003, Wang Jun crée une entreprise avec son cousin, curieusement, il ne fabrique pas des chemises comme il a appris à le faire au cours des

7 À cette époque, Yiwu apparaît comme le lieu où ont pu être accumulées des ressources autorisant un retour dans le pays d'origine. 
années précédentes, mais des foulards. L'explication qu'il donne tient aux coûts de production : moins complexe à fabriquer, le produit nécessite un investissement moins important. Le succès de sa première collection tient aussi à une innovation : ses foulards ne sont pas de simples morceaux de tissus imprimés, il fixe sur ses derniers des accessoires de bijouterie et c'est cette idée qui lui assure un succès rapide auprès de clients étrangers (Wang, 2009).

Cette flexibilité, dans le passage d'un produit à un autre, du commerce à la production, du travail salarié au travail indépendant, ne peut se comprendre que dans le contexte d'un marché en croissance rapide et où les opportunités sont nombreuses et se renouvellent rapidement. Pour ne parler que du marché du travail, cela fait maintenant plusieurs années que les entreprises du Zhejiang ont des difficultés à recruter des travailleurs migrants, notamment après le nouvel an chinois, période de renouvellement des contrats de travail. À condition d'être prêts à réviser à la baisse ses prétentions salariales et ses conditions de travail, les migrants trouvent donc toujours à s'employer. Par ailleurs, le marché d'Yiwu a connu une croissance continue au cours des vingt dernières années, la clientèle internationale (Asie du Sud, Moyen-Orient, Afrique) étant toujours plus nombreuse.

Si les parcours professionnels des acteurs rencontrés sont marqués par la flexibilité, celle du frère, à la tête d'une entreprise prospère de plus de 100 salariés, se distingue par sa cohérence. Sa réussite va de pair avec une stabilité dans sa vie personnelle alors que les deux sœurs ont vécu des conflits familiaux et ont connu de sérieux problèmes de santé. Wang Jun est d'ailleurs le seul à organiser son récit autour de l'apprentissage d'un métier et de l'acquisition de compétences, à la différence de ses sœurs. II insiste sur son apprentissage initial qui lui a permis de progresser dans les métiers de la confection : coudre des ourlets, démonter et remonter une machine à coudre, coudre des cols de chemise, puis concevoir un patron. II a dû cependant faire preuve de ténacité car les tailleurs sont réticents à former un jeune homme qui peut devenir leur concurrent. II s'est contenté tout d'abord d'apprendre dans des manuels, puis il est parvenu à devenir l'apprenti officieux d'un tailleur - il apprend en l'observant, le seconde sans être rémunéré, et apprend la coupe le soir après ses heures de travail. Quand le récit du frère est celui d'une marche progressive vers la maîtrise d'un métier, celui des deux sœurs est le témoignage d'une lutte permanente pour maintenir une activité. Shi Lu (2013) avance une explication en termes de genre pour comprendre les inégalités professionnelles. Dans notre étude de cas, il ressort que les deux sœurs de Wang Jun ont été moins soutenues que leur frère dans leurs projets entrepreneuriaux, et qu'elles ont rencontré de multiples obstacles de la part de leur famille et belle-famille.

\section{Les formes de la mobilité}

Un des ressorts communs à l'activité entrepreneuriale de nos trois personnages est la façon dont ils jouent sur les ressources des espaces économiques à I'intérieur du territoire chinois (Guiheux, 2004), Yiwu se trouvant entre les zones de production du Sud du pays les plus connectées au marché international et la Chine de l'intérieur où la main-d'œuvre est moins chère et la demande moins exigeante. La mobilité, à la recherche de fournisseurs moins chers ou de 
modèles nouveaux, apparaît comme l'une des caractéristiques structurantes de leur activité et de leurs itinéraires.

Ainsi Wang Yan qui alors qu'elle est commerçante, souhaite se rendre à Shishi, au sud de la province du Fujian, où existe un marché spécialisé dans les vêtements pour enfants. Son intention est, dit-elle de "comprendre le marché " (liaojie shichang), mais il s'agit plutôt de repérer les produits qui se vendent bien pour ensuite les faire reproduire et approvisionner le marché d'Yiwu. À une autre occasion, elle se rend dans un marché de Fuzhou (Fujian) pour le même motif où elle visite des dizaines de producteurs. En 2003, pendant la crise du $\mathrm{Sras}^{8}$, alors que ses clients sont de moins en moins nombreux et que ses stocks augmentent, elle estime qu'il lui faut renouveler ses produits et se rend à Canton à la recherche de nouveautés. Sur place, elle rencontre un patron originaire du Jiangxi, elle lui dissimule son activité de commerçante et obtient quelques échantillons qu'elle fera ensuite fabriquer dans le Hunan, la province d'origine de son mari où aujourd'hui, plusieurs autres marchands d'Yiwu s'approvisionnent.

La mobilité permet de découvrir un nouveau produit ou un nouveau lieu de fabrication qui assurera temporairement un avantage compétitif dans un univers extrêmement concurrentiel.

Les mobilités dans cette économie du prêt-à-porter sont de plusieurs ordres. Ce sont d'abord les personnes qui se rendent d'un point à un autre pour découvrir de nouveaux produits, de nouvelles modes (et ainsi être en avance sur leurs concurrents) ou de nouveaux lieux de production moins onéreux. Ce sont les modèles qui circulent, en particulier du Sud de la Chine, la plus connectée aux marchés internationaux, vers l'intérieur. Ce sont aussi les articles eux-mêmes confectionnés dans une province pour être revendus dans une autre. Bien que ces marchands fassent tous usage des technologies de l'information qui leur permettent d'être en permanence en contact avec les fournisseurs et les clients en Chine et dans le reste du monde, ils se déplacent physiquement et sont à l'affût des opportunités qu'offrent les différences de coûts ou le décalage temporel dans la diffusion des modes. À cet égard, ces entrepreneurs migrants du prêt-à-porter ont des points communs avec les entrepreneurs "nomades" (Tarrius, 2002 ; Peraldi, 2002), à la différence notable que les mobilités dont nous parlons ne se limitent pas aux contacts maintenus avec la province d'origine (ici le Jiangxi) et que leurs activités ne relèvent pas du secteur informel.

\section{Conclusion}

Yiwu, comme de nombreuses villes côtières, accueille de nombreux travailleurs de l'extérieur, venus de la Chine rurale et qui aspirent à une mobilité sociale ascendante. Ville marchande et manufacturière, Yiwu donne peut-être plus qu'ailleurs aux migrants la possibilité de jouer sur les activités de production et de commercialisation.

Le prêt-à-porter ouvre d'abord nombre d'opportunités pour des raisons structurelles. À Yiwu comme ailleurs dans le monde, la production est organisée

8 Syndrome respiratoire aigu sévère. 
de manière pyramidale avec au sommet des entreprises créatives et commerciales et à la base des ateliers qui réalisent la marchandise. Bien que la concurrence soit âpre et oblige à minimiser les coûts, les conditions de production donnent lieu à d'importantes créations d'entreprises. Même dans le cas d'une production bas de gamme destinée à des acheteurs pauvres, les itinéraires que nous avons relatés sont ceux d'individus qui ont été ouvriers, puis à la tête de petits ateliers avant de développer une activité commerciale où la marge est plus élevée. À leur échelle, les deux sœurs conçoivent - en fait copient - les produits qu'elles vendent, qui sont fabriqués dans des ateliers de sous-traitance à Yiwu ou ailleurs.

De leur côté, les travailleurs de l'extérieur possèdent toute une série de ressources propres, favorisant l'accès à l'entrepreneuriat, qualifiées dans $d^{\prime}$ autres contextes de ressources " ethniques ${ }^{9}$. Si ces acteurs disposent de peu de capital, d'un niveau d'éducation faible ou n'ont aucune connexion avec le pouvoir politique local - ressources que possèdent les entrepreneurs locaux -, ils peuvent prendre appui sur leur famille - parenté restreinte ou élargie - ou leur communauté d'origine pour réunir le capital nécessaire pour débuter une activité indépendante, ou disposer d'un réseau de fournisseurs et de clients. La littérature sur les enclaves ethniques a montré comment les migrants peuvent ainsi développer des stratégies malgré leur position d'outsiders (Waldinger, Aldirch et Ward, 1990). Dans les trois cas analysés, les opportunités ont été d'autant plus importantes que la fratrie est arrivée à Yiwu alors que le bourg, encore rural, était en pleine phase de décollage économique. Les liens de parenté avec des locaux ont contribué à leur installation. Un travailleur rural arrivant à Yiwu sans ressource sociale ni compétence professionnelle n'aurait pas aujourd'hui les mêmes chances. Les dispositifs évoqués qui font que les populations de l'extérieur sont moins discriminées à Yiwu que dans les plus grandes métropoles chinoises ont également joué leur rôle.

L'originalité du cas d'Yiwu tient à sa position dans l'échelle des marchés mondiaux du prêt-à-porter. Les marchandises produites et échangées sont destinées à une clientèle à faible pouvoir d'achat en Chine et dans le reste du monde. Cela autorise les entrepreneurs à jouer de leur position intermédiaire : en copiant des produits labellisés de meilleure qualité, en les faisant fabriquer à moindre coût et sans marque, ils permettent, in fine, aux consommateurs pauvres de participer aux circulations d'une industrie de la confection mondialisée.

Alors que la vie professionnelle des deux sœurs semble s'être déroulée sous le sceau de la précarité, celle du frère marquée pourtant par des à-coups et des changements, se présente comme un parcours régulier vers sa réussite actuelle. À la différence de ses sœurs dont l'activité principale est commerciale et de petite taille, Wang Jun est à la tête d'une entreprise moyenne comptant plus d'une centaine de salariés qui produit et écoule directement une partie de sa marchandise auprès de clients étrangers. Comment expliquer que ce dernier,

9 Ce qui est " ethnique " à propos de l'économie, du marché du travail ou de la concentration des commerces " ne peut être rien de plus qu'un ensemble de connexions et d'échanges réguliers entre des personnes partageant une origine nationale ou des expériences de migrations semblables " (Waldinger, 1993 : 18). 
davantage que ses sœurs, ait pu acquérir les compétences nécessaires à la consolidation de son activité ? Ce que révèle l'analyse sur la base de récits de $v^{i} e^{10}$, tient aussi à l'importance de la dimension personnelle. Ce qui distingue la trajectoire du frère de celle de ses deux sœurs, c'est qu'il a été le seul à avoir pu et su s'approprier certaines ressources et acquérir les compétences lui permettant d'exercer un contrôle sur sa carrière. Nous rejoignons ici une conclusion à laquelle nous parvenions à l'échelle d'un autre terrain, celui de métiers urbains de service à Shanghai (Guiheux et Zalio, 2010). Comme nous l'écrivions ailleurs, " il ne suffit pas qu'il existe des opportunités, encore faut-il que l'acteur s'en saisisse. Les récits biographiques permettent de saisir comment les individus mettent en place des stratégies appropriées et montrent comment les migrants peuvent prendre le contrôle de leur propre vie sur la base d'une expérience accumulée et d'une motivation pour réaliser l'objectif d'être son propre patron " (Guiheux et Zalio, 2013).

\section{Références bibliographiques}

Bretell Caroline B. and Alstatt Kristoffer E. (2007) The Agency of Immigrant Entrepreneurs. Biographies of the self-employed in ethnic and occupational niches of the urban labor market, Journal of Anthropological Research, 63, pp. 383-397.

Chan Kam Wing (2010) The Household Registration System and Migrant Labor in China: Notes on a Debate, Population and Development Review, 36 (2), pp. 357-364.

Chin Margaret M. (2005) Sewing Women: Immigrants and the New York City Garment Industry, New York, Columbia University Press, 197 p.

Froissart Chloé (2008) Le système du hukou : pilier de la croissance chinoise et du maintien du PCC au pouvoir, Les études du Ceri, 149, 47 p.

Green Nancy L. (1998) Du Sentier à la 7e avenue. La confection et les immigrés Paris-New York, 1880-1980, Paris, Seuil, 461 p.

Guiheux Gilles (à paraître) Yiwu, Chine : Bourg rural devenu place marchande mondialisée, présenté au colloque " Entre le licite et l'illicite : migrations, travail, marchés ", Cerisy, 12-18 septembre 2011.

Guiheux Gilles (2009) L'entrepreneur, héros socialiste, Revue Espaces Marx, 26, pp. 52-56.

Guiheux Gilles (2005) Le renouveau du capitalisme familial. Défense et illustration par un entrepreneur du Zhejiang, Perspectives chinoises, 87, pp. 22-32.

Guiheux Gilles (2004) Les ressources localisées d'une entreprise privée en Chine centrale, Genèses. Sciences sociales et histoire, 56, pp. 51-71.

Guiheux Gilles et Zalio Pierre-Paul (à paraître) Entrepreneuriat et migration, in Michel Grosseti et Pierre-Paul Zalio, Lexique sociologique de l'activité entrepreneuriale, Paris, Presses de Sciences po.

10 Voir Kontos, 2003 ; Bretell et Alstatt, 2007. 
Guiheux Gilles et Zalio Pierre-Paul (2010) Agents immobiliers à Shanghai. Des carrières de migrants à col blanc, Perspectives chinoises, 4, pp. 52-63.

Kontos Maria (2003) Considering the concept of entrepreneurial resources in ethnic business: Motivation as a biographical resource?, International Review of Sociology, 13, pp. 183-204.

Li Raymond (2012) Hukou reform plan enters final stage. New system of residence permits will be submitted to the State Council for approval within a year, South China Morning Post, 12/03/2012.

Mu Guo (2010) The Yiwu Model of China's Exhibition Economy, Provincial China, 2-1, pp. 91-115.

Peraldi Michel (Éd.) (2002) La fin des norias. Réseaux migrants dans les économies marchandes en Méditerranée, Paris, Maisonneuve et Larose, 495 p.

Shi Lu (à paraître) Figure de migrants - Itinéraire et trajectoire d'une commerçante au Zhejiang, Travail, genre et sociétés.

Tarrius Alain (2002) La mondialisation par le bas. Les nouveaux nomades de I'économie souterraine, Paris, Balland, $168 \mathrm{p}$.

Waidiren de zhanling zhongguo zuida yemin xian Yiwu de wailai renkou guanli kunju (2011) (Les dilemmes de la gestion de la population migrante à Yiwu, le xian de Chine où la population migrante représente la part la plus importante), Fenghuang zhoukan, 04/08/2011, [en ligne] consulté le 30/04/2012. URL : http:// news.ifeng.com/fhzk/detail_2011_08/04/8176640_0.shtml

Waldinger Roger (1993) Le débat sur I'enclave ethnique : revue critique, Revue Européenne des Migrations Internationales, 9 (2), pp. 15-29.

Waldinger Roger (1986) Through the Eye of the Needle: Immigrants and Enterprise in New York's Garment Trades, New York, New York University Press, $231 \mathrm{p}$.

Waldinger Roger (1984) Immigrant Enterprise in the New York Garment Industry, Social Problems, 32 (1), pp. 60-71.

Waldinger Roger, Aldrich Howard and Ward Robin (1990) Ethnic Entrepeneurs: Immigrant Business in Industrial Societies, Londres, Sage, 226 p.

Wang Xingliang (2009) Yi tiao weijin, rang dagongzi kaiqi yuma (Un foulard qui permet à un travailleur migrant d'être aujourd'hui au volant d'une BMW), Yiwu shangbao, 13/12/2009, [en ligne] consulté le 29/03/2012. URL : http://pdf.ywnews. cn/2009/12/13/sb1.pdf

Wu Yuehua (2007) [Wang Jun]: ba weijin maidao oumei qu ([Wang Jun] : vendre des foulards jusqu'en Amérique et en Europe), Shangrao ribao, 18/06/2007, [en ligne] consulté le 30/04/2012. URL : http://gov.srzc.com/html/20076/18/1893103834.htm

Xin huangyuan shichang zai zhen Yiwu fuzhuang ye xiongfeng (2011) (Le nouveau marché Xinhuang restitue son lustre au prêt-à-porter de Yiwu), Yiwu shangbao, 02/07/2011, [en ligne] consulté le 26/04/2012. URL : http://mlzj.zjol. com.cn/mlzj/system/2011/07/02/013943333.shtml

Yiwu wailai renkou shouci tubo 150 wan (2012) (Pour la première fois à Yiwu, la population venue de l'extérieur dépasse 1,5 million), Jinhua wanbao, 14/01/2012, [en ligne] consulté le 13/07/2012. URL : http://jhnews.com.cn/jhwb/2012-01/14/ content_2041010.htm 
Yiyang xian tuijin 'keshang da zoufang, xiangma da duijie' huodong (2011) (Le xian deYiyang entreprend des visites pour promouvoir l'activité et accueillir de grands projets), site du gouvernement de la ville de Shangrao, 25/08/2011, [en ligne] consulté le 30/04/2012. URL : http://www.srsw.gov.cn/Read.Asp?PPNewsID=433

Yiyang xian zhaoshang yinze gongzuozhong de 'bianzhengfa' (2010) (la « dialectique " du travail d'attraction d'investissements dans le xian de Yiyan), site de la ville de Yiyang, 03/06/2010, [en ligne] consulté le 30/04/2012. URL : http://www. jxyy.gov.cn/content/2010/06/03/5813.htm

Zhou Min (1992) Chinatown: The Socioeconomic Potential of an Urban Enclave, Philadelphia, Temple University Press, 275 p.

Zheng Guangquan ludui kaizhan 'Zhejiang qianli hang' zhaoshang huodong (2011) (Zheng Guangquan conduit une délégation pour mobiliser les investisseurs dans le cadre du mot d'ordre " des milliers de kilomètres dans le Zhejiang "), site du gouvernement local du xian de Yugan, 28/10/2011, [en ligne] consulté le 27/04/2012. URL : http://www.yugan.gov.cn/jryg/2011/10/28/5438.htm 


\section{Gilles Guiheux}

\section{Travailleurs migrants du prêt-à-porter en Chine. Flexibilités et opportunités}

Cette contribution questionne les conditions de production du prêt-à-porter en Chine et la place qu'y occupent les travailleurs migrants. II ne s'agit pas de migrants internationaux mais de migrants internes venus des provinces rurales pauvres. Ils partagent avec les premiers un certain nombre de caractéristiques liées à leur désir d'ascension sociale et aux discriminations dont ils sont victimes. L'hypothèse est que, bien qu'en Chine comme ailleurs, les travailleurs migrants du prêt-à-porter travaillent dans des conditions difficiles pour des salaires faibles, ils ne sont pas simplement les victimes passives d'un système d'emploi oppressif. Cette contribution s'appuie sur la trajectoire biographique et professionnelle des membres d'une fratrie, rencontrée à Yiwu, ville marchande et manufacturière de la province du Zhejiang. Leurs parcours socio-professionnels sont rapportés aux caractéristiques du secteur fait d'une multitude de petites et moyennes entreprises familiales.

\section{Migrant Workers and the Garment Industry in China. Flexibilities and Opportunities}

This article questions the conditions of production in the Chinese garment industry and the role played by migrant workers. There are not international migrants but internal migrants coming from poor rural areas to more advanced cities. They share with the former a desire for upward social mobility and their exposure to discriminations. The paper argues that, although in China as elsewhere, garment workers work in difficult conditions for low wages, they are not simply passive victims of an oppressive employment system. This contribution is based on the trajectory of three siblings, met in Yiwu, Zhejiang province. Their professional paths are related to the characteristics of the industry, composed of a multitude of small and medium-sized family businesses.

\section{Trabajadores migrantes del pronto a vestir en China. Flexibilidades y oportunidades}

Este artículo cuestiona las condiciones de producción del pronto a vestir en China y el lugar que ocupan en esta producción los trabajadores migrantes. No se trata de migrantes internacionales, sino de migrantes internos oriundos de provincias rurales pobres. Ellos comparten con migrantes internacionales una serie de características relacionadas con su deseo de movilidad social ascendente y la discriminación que sufren. El supuesto es que, aunque, en China como en otros lugares, los trabajadores migrantes del pronto a vestir trabajan en condiciones difíciles por salarios bajos, ellos no son simplemente víctimas pasivas de un sistema de empleo opresivo. Esta contribución se basa en la trayectoria biográfica y profesional de hermanos encontrados en Yiwu, ciudad comercial y manufacturera de la provincia de Zhejiang. Sus trayectorias socioprofesionales están relacionadas con las características del sector de actividad, constituido por una multitud de empresas familiares de pequeño y medio porte. 